\title{
VENTILAORY CHANGES AFTER INSPIRATORY MUSCLE TRAINING IN CHRONIC HEART FAILURE PATIENTS
}

\author{
By \\ ${ }^{1}$ Helmy ZM, ${ }^{2}$ IbrahimBS, ${ }^{1}$ Mohammed SH, ${ }^{3}$ Mohammed RA, ${ }^{4}$ HassanMH \\ ${ }^{1}$ Department of Physical Therapy for Cardiovascular / Respiratory Disorder and Geriatrics. Faculty \\ of Physical Therapy, Cairo University. ${ }^{2}$ Department of Cardiology, ${ }^{3}$ Department of Clinical Pathology, \\ ${ }^{4}$ Department of Physical Therapy, National Heart Institute, Cairo.
}

\begin{abstract}
Introduction: inspiratory muscle training (IMT) improves exercise capacity and ventilatory responses to exercise in $\mathrm{CHF}$ patients with inspiratory muscle weakness (IMW). Aim of Work: to investigate the effect of inspiratory muscle training (IMT) with comprehensive cardiac rehabilitation (CR) program (aerobic and resisted exercise training) on ventilatory changes in chronic heart failure patients. Materials and Methods: Forty eligible male patients with chronic heart failure secondary to ischemic heart disease but only thirty of them completed the study. Their ages ranged from 50-60 years old .They were randomly assigned to 6 months of aerobic exercise, resisted exercise plus IMT $(n=15)$ or to aerobic exercise plus resisted exercise only $(n=15)$, Before and after intervention, the following measures were obtained: maximal inspiratory muscle pressure (PImax), ventilatory equivalent of $\mathrm{CO}_{2}$ at anaerobic threshold of cardiopulmonary exercise testing. Result: Compared aerobic exercise (AE) + IMT to AE resulted in additional significant difference in $\mathrm{EqCO}_{2}(25.43 \%$ vs $16.77 \%$ ) and in $\mathrm{PI}_{\max }(14.81 \%$. vs $34.94 \%$.). Conclusion: This study demonstrates that the addition of IMT to AE results in improvement in ventilator responses to exercise in selected patients with CHF and IMW
\end{abstract}

Key words: Inspiratory muscle training, Chronic heart failure, Maximal inspiratory pressure and Aerobic exercise . 


\section{Introduction}

Heart failure (HF) is a complex clinical syndrome manifesting as inability to supply adequate blood flow throughout the body due to any structural or functional cardiac abnormality (Bosnak-Guclu et al., 2011).

The most common complaints are exercise intolerance, dyspnea, and fatigue in patients with heart failure. Mechanisms are multifactorial and interacting each other in patients with heartfailure. Dominant catabolic process caused by left ventricular impairment, leads to respiratory and skeletal muscle myopathy. Increased ergoreflex activation caused by myopathy results in exercise intolerance. Fatigue is a frequent symptom among $\mathrm{HF}$ due to impaired cardiac pump is responsible for inadequate oxygen delivery and build up waste products from anaerobic metabolism (Bosnak-Guclu et al., 2011).

Respiratory muscle dysfunction may play a role in limiting exercise capacity; it has been proposed that dyspnea is influenced by the central nervous system's perception of inspiratory motor output, a signal that increases with a reduction in respiratory muscle strength. The maximal inspiratory pressure and maximal expiratory pressures, as well as respiratory muscle endurance, are reduced in patients suffering from $\mathrm{CHF}$ compared with age-matched normal subjects. This reduction correlates with the degree of dyspnea (Ribeiro et al., 2009 and Thayse Lucena et al., 2012).

Some of these patients show reduced maximal inspiratory pressure and endurance of inspiratory muscles. Abnormal ventilator response to exercise, periodic breathing, and delayed oxygen uptake during recovery of maximal effort have also been associated with severity and poor prognosis in CHF. Patients with chronic CHF have decreased lung volume, decreased compliance, increased airway closing pressure, increased work of breathing, and greater oxygen consumption. All of these are recognized as additional factors implicated in the limited exercise response, as well as in poor prognosis. Pulmonary rehabilitation may improve quality of life and exercise capacity in patients with chronic heart failure (Badr et al ., 2014).

Selective inspiratory muscle training is effective in patients with chronic heart failure Mechanisms underlying these beneficial effects of IMT included attenuated metaboreflex, 
improved ventilatory efficiency, and lower ventilatory oscillations during incremental exercise. It is proposed that the metabolic products accumulated from fatiguing respiratory muscle contraction could increase sympathetic vasoconstriction activity (the inspiratory muscle metaboreflex), and the attenuation of the metaboreflex could then improve blood flow redistribution to skeletal muscles in the body, thereby delaying the time to fatigue and decreasing workload on the heart . IMT may decrease work of breathing, metabolic costs of breathing and perception of nervous system's perception of inspiratory output, so that dyspnea and exercise capacity would be improved (Wong et al., 2011)

(Stein et al., 2009) shown that inspiratory muscle training (IMT) improves exercise capacity and ventilatory responses to exercise in CHF patients with inspiratory muscle weakness (IMW).

\section{Aim of work}

To investigate the effect of inspiratory muscle training (IMT) with comprehensive CR program (aerobic and resisted exercise training) on ventilatory changes in chronic heart failure patients.

\section{Materials and Methods}

Study design: cross sectional study

Place and duration of the study: the study was carried out in National Heart Institute outpatient clinic. During the period from March 2013 till July 2014

Study sample: Forty eligible male patients with chronic heart failure secondary to ischemic heart disease and only thirty of them completed the study. They were diagnosed by echocardiography and coronary angiography. Their ages ranged from 50-60 years old .The patients had been clinically and medically stable for more than three months prior to the onset of study period.

Inclusion criteria included:- The diagnosis of ischemic cardiomypathy, they were on optimal medical therapy with no major changes in treatment regimen during the study, left ventricular end-diastolic dimension $>5.5 \mathrm{~cm}$ and end-systolic diameter $>$ $4.5 \mathrm{~cm}$, Fractional shortening $<25 \%$ and Ejection fraction $<40 \%$. New York Heart Association (NYHA) class II-III.

Study methods: They was randomly divided into two groups equal in number and age matched, similarity 
of both groups should be accurate with respect to age, weight, height and body mass index (BMI). All participants were informed about the nature and effects of trial. They have been given an informed written consent form.

\section{Group (A) (Training group):} Fifteen patients participated in a supervised comprehensive CR rehabilitation program which is composed of: Aerobic training: in form of Interval aerobic training: Strengthening exercise, Resistance/ strength training, Inspiratory muscle trainer. The training subjects received information regard the benefits of regular aerobic exercise and were asked to report any side effects during the treatment session.

\section{Group (B): (Control group):} Fifteen patients participated in comprehensive aerobic training: in the form of Interval aerobic training and Strengthening exercise: Resistance/ strength training.

\section{Instrumentation:}

1. For assessment: Oxycon pro (ER900, Ergoline, Jaeger, Würzburg, Germany) cardiopulmonary exercise test unit with 12 channel ECG, gas analyzer to measure maximal $\mathrm{O}_{2}$ uptake and anaerobic threshold, Mercurial sphygmomanometer is used to for measuring blood pressure before, during, and after exercise training sessions, Free weights for assessing maximum voluntary contraction by one repetition maximum test before conducting the circuit weight training for both groups , Maximum Inspiratory Pressure meter (care fusion UK 2321td) to determine the inspiratory muscle strength before and after training in both groups.

2. For training: Inspiratory muscle trainer: (Threshold inspiratory muscle trainer; Respironics, Cedar Grove, New Jersey 07009-1201 USA). Used for training the study group. The device contains a spring-loaded valve, attached to a mouthpiece through which subjects inspire while wearing a nose clip. The valve controls a constant inspiratory pressure training load and the patient must generate the inspiratory pressure in order for the inspiratory valve to be opened and allow inhalation of air. The amount of resistance can be adjusted by varying the compression of the spring-loaded valve. Adjustment 
from $7 \mathrm{cmH}_{2} \mathrm{O}$ to $41 \mathrm{cmH}_{2} \mathrm{O}$ is possible (Crowe et al., 2008).

\section{Procedure:}

\section{Evaluation procedure:}

II- Cardiopulmonary exercise testing (CPET):

The equipment consists of a metabolic cart and a static cycle/ treadmill. The metabolic cart contains gas analyser, a computer and screens which display continuously 12lead ECG ST segment analysis and graphical displays of the physiological changes as they occur during exercise. The gas analyzers are capable of breathby breath measurement of oxygen consumption $\left(\mathrm{VO}_{2}\right)$ and carbon dioxide production $\left(\mathrm{VCO}_{2}\right)$ and flow calibration is done before each test. The test is performed in an adequately ventilated room with all resuscitation facilities (Agnew., 2010).

After a period of rest to allow the patient to become familiar with the equipment and the bicycle, the resting Heart Rate (HR), Blood Pressure (BP), $\mathrm{SpO}_{2}, \mathrm{ECG}$ and gas exchange values are recorded. In a cycle ergometer the saddle height is adjusted and patients advised to pedal at constant speed of 50 to $60 \mathrm{rpm}$ with monitors attached and the tight-fitting face mask in place seal over the patients' nose and mouth to prevent air leakage, with no resistance for a short period of 2 to 3 minutes. Then work rate is increased by 10 to 20 W. min- 1 by the computer, increasing the resistance of the pedals (bicycle) while the subject maintains a constant pedaling rate (bicycle). . All patients were subjected to a submaximal exercise testing on stationary ergometry of the cardiopulmonary exercise test before and after training programs according to Ramp protocol. The optimal duration of the test is around ten minutes for proper assumption of the $\mathrm{VO}_{2} \max$. The maximum aerobic capacity i.e. $\mathrm{VO} 2 \mathrm{max}$ is the highest $\mathrm{VO}_{2}$ recorded when a patient's $\mathrm{VO}_{2}$ value reaches a plateau with work rate increments and are based on predetermined formulae using the patients age, height, gender and weight .Exercise finishes with a cool down stage in which the patient pedals the bicycle for a brief period against zero resistance (Chatterjee et al., 2013).

\section{Maximum inspiratory pressure test :}

Maximum inspiratory pressure was performed for both patients groups for assessing inspiratory muscle strength pre and post the training program and 
to identify the training load of the inspiratory muscle trainer. The test was explained to the patients before applying. Measurement was obtained in the sitting position, the patients asked to expire all the air inside out of the device then put the mouthpiece while noise clip applied and inspire as rapid as they can and hold for at least 1.5 seconds, so that the maximum pressure could be recorded. The patients were asked to repeat the technique for 3 times with sufficient resting periods in between so that they could reach their maximum performance (winkelmann et al., 2009).

\section{Training procedure:}

Both groups of patients performed a supervised individual training program based on the result of cardiopulmonary exercise testing, Both groups were trained using heart rate range or reserve method (Karvonen's method); training heart rate $(\mathrm{THR}=\mathrm{HR}$ rest + (HRmax - HRrest) $55-85 \%$ This formula is the most accurate one ( Kennedy et al .2012)

Mode of exercise: treadmill and cycle ergometer.

- Intensity of exercise: the patient should not exceed his training heart rate during exercise period. The training heart rate increased gradually according to each patient's response during exercise training session, starting with $55 \%$ of heart rate reserve, till trying to reach $85 \%$ at the end of $6^{\text {th }}$ month.

- Type of exercise: The conditioning phase of each session involved aerobic training (cycle ergometer, treadmill walking) and resistance/strength training.

Aerobic training: at the prescribed heart rate intensity. All subjects used a heart rate monitor (Telemetry, Hewlett Packard (HP) M2604A) to obtain the assigned exercise intensity. The Borg 6-to-20 scale used to assess the rate of perceived exertion during and after each training session. . Patients in both groups exercised continuously for 45 minutes without breathing heavily, the progression of exercise training appeared as the number of bouts along $45 \mathrm{~min}$ of exercise training decrease gradually until it can reach at end of 6 months to no rest with one bout of training.

\section{Resistance/strength}

training (RST): Consists of eight resistance exercises for different eight large muscle groups (shoulder flexors and extensors, elbow flexors and extensors, 
hip flexors and extensors and knee flexors and extensors). It was applied for both groups of patients.

RST can be started with a high number of repetitions (12-25) and a low intensity (30-40\% 1-RM), When the patient is confident with the exercise, he can proceed to the next phase.

As RST at higher intensity become (40-60\% 1-RM) in order to increase muscle mass after 2 months. Repetitions were slowly increased from 1 _ 10,1 _ $15,2_{\text {_ }} 10$ to $2_{\text {_ }} 15$. Between each series of repetitions $1 \mathrm{~min}$ rest was allowed. Patients were instructed in correct lifting techniques, to avoid Valsalva manoeuvre. So intensity will be detected initially, by increasing the number of exercise circuits from one to three, followed by increasing the resistance or cycling load (Piepoli et al., 2011).

\section{-Duration: each exercise session included three phases:}

Warm up phase: an initial 5-10 minutes in the form pedaling on bicycle ergometer, walking on treadmill or active stretching exercises with breathing. The heart rate during warmup phase reached $30-40 \%$ of the target heart rate.
Aerobic phase: at the prescribed heart rate intensity. This phase started in short bouts about 8 minutes for 24 minutes, gradually prolonged up till continuous 45 minutes at the end of the 7th months.

Cool down phase: for 5-10 minutes with intensity decreased gradually to resting heart rate.

Frequency: exercise training done three times per week for six months.

Patients in group A (study group) performed the breathing exercise with the inspiratory muscle trainer for 7 sets with an inspiratory load at $30 \%$ of PImax, three time per week for six months ; training loads were adjusted to maintain $30 \%$ of the PImax (Winkelmann et al, 2009).

The patients were taught the following instructions for proper inspiratory muscle training:

- Sit in a comfortable position and put the nose clip on the nose, inhale through the mouth only.

- Relax, place the lips around the mouthpiece, and inhale as deeply as you can with enough force to open the valve. 
- Exhale through the mouthpiece; continue inhaling and exhaling without removing the device from the mouth.

- Repeat inhaling 7 sets every set 3 minutes with enough resting period's in-between sets (1min). Total time of training 28 minutes three time per week.

\section{Data management:}

- Descriptive statistics and t-test for comparison of the mean age, weight, height, BMI and duration of infarction, EF, FS between both groups (study and control).

- Mixed ANOVA was conducted to compare the effect of time (pre versus post) and the effect of treatment (between groups), as well as the interaction between time and treatment on mean values of measured variables.

- The level of significance for all statistical tests was set at $\mathrm{p}<0.05$.
- All statistical measures were performed through the statistical package for social studies (SPSS) version 19 for windows.

\section{Consent:}

Authors declare that verbal consent was taken from the studied group before making the study.

\section{Ethical approval :}

The ethical committee of faculty of physical therapy approved the study protocol .

\section{Results}

Comparing the subject demographic and clinical characteristics data of both groups revealed that there was no significance difference between both groups $(\mathrm{p}>0.05)$.

A-Comparsion of ventilatory equivalent of $\mathrm{CO}_{2}$ at anaerobic threshold ( $\mathrm{EqCO}_{2}$ at $\left.\mathrm{AT}, \mathrm{ml} / \mathrm{min}\right)$ of cardiopulmonary exercise testing between both groups : 
Table 1: Mean values of $\mathrm{EqCO}_{2}$ pre and post treatment of study and control groups:

\begin{tabular}{|c|c|c|c|c|}
\hline \multicolumn{5}{|c|}{$\mathrm{EqCO}_{2}(\mathrm{ml} / \mathrm{min})$} \\
\hline \multicolumn{2}{|c|}{ Study group } & \multicolumn{3}{|c|}{ Control group } \\
\hline \multicolumn{2}{|c|}{$\bar{X} \pm S D$} & \multicolumn{3}{|c|}{$\overline{\mathrm{X}} \pm \mathrm{SD}$} \\
\hline Pre treatment & Post treatment & \multicolumn{2}{|c|}{ Pre treatment } & Post treatment \\
\hline $44.51 \pm 8.36$ & $33.19 \pm 4.05$ & \multicolumn{2}{|c|}{$45.79 \pm 5.17$} & $38.11 \pm 3.89$ \\
\hline \multicolumn{5}{|c|}{ Within group comparison } \\
\hline & & MD & $\begin{array}{c}\% \text { of } \\
\text { improvement }\end{array}$ & p-value \\
\hline \multirow{2}{*}{ Pre test vs. post test } & Study group & 11.32 & $\downarrow 25.43$ & $0.0001 *$ \\
\hline & Control group & 7.68 & $\downarrow 16.77$ & $0.0001 *$ \\
\hline \multicolumn{5}{|c|}{ Between group comparison } \\
\hline & & & MD & p-value \\
\hline \multirow{2}{*}{ Study vs control } & Pre & & 1.28 & 0.68 \\
\hline & Post & & 4.92 & $0.01 *$ \\
\hline
\end{tabular}

$\overline{\mathrm{X}}$ : Mean SD: Standard Deviation

p value: Probability value
MD: Mean difference

*: Significant

Table (1) showed that there was a significant decrease in the mean values of $\mathrm{EqCO} 2$ post treatment of the study group compared to the control group $(\mathrm{p}=0.01)$.

B-Comparsion of maximal inspiratory pressure (PImax) between both groups 
Table 2: Mean values of PImax pre and post treatment of study and control groups:

\begin{tabular}{|c|c|c|c|c|}
\hline \multicolumn{5}{|c|}{$\operatorname{PImax}\left(\mathrm{cmH}_{2} \mathrm{O}\right)$} \\
\hline \multicolumn{2}{|c|}{ Study group } & \multicolumn{3}{|c|}{ Control group } \\
\hline \multicolumn{2}{|c|}{$\overline{\mathrm{X}} \pm \mathrm{SD}$} & \multicolumn{3}{|c|}{$\bar{X} \pm \mathrm{SD}$} \\
\hline Pre treatment & Post treatment & \multicolumn{2}{|c|}{ Pre treatment } & Post treatment \\
\hline $84.7 \pm 16.68$ & $114.3 \pm 17.85$ & \multicolumn{2}{|c|}{$81.7 \pm 9.71$} & $93.8 \pm 11.82$ \\
\hline \multicolumn{5}{|c|}{ Within group comparison } \\
\hline & & MD & $\begin{array}{c}\% \text { of } \\
\text { improvement }\end{array}$ & p-value \\
\hline \multirow{2}{*}{ Pre test vs. post test } & Study group & -29.6 & $\downarrow 34.94$ & $0.0001^{*}$ \\
\hline & Control group & -12.1 & $\downarrow 14.81$ & $0.0001^{*}$ \\
\hline \multicolumn{5}{|c|}{ Between group comparison } \\
\hline & & & MD & p-value \\
\hline \multirow{2}{*}{ Study vs control } & Pre & & 3 & 0.62 \\
\hline & Post & & 20.5 & $0.007 *$ \\
\hline
\end{tabular}

$\overline{\mathrm{X}}$ : Mean SD: Standard Deviation

$\mathrm{p}$ value: Probability value
MD: Mean difference

*: Significant

Table (2) showed that there was a significant increase in the mean values of PImax post treatment of the study group compared with control group $(\mathrm{p}=0.007)$. 


\section{Discussion}

With regard to the comparison between the two groups the results of the present study revealed significant difference between both groups in $\mathrm{EqCO}_{2}$ - ventilatory equivalent of $\mathrm{CO}_{2}$ at anaerobic threshold $\left(\mathrm{EqCO}_{2}\right.$ at $\mathrm{AT}$, $\mathrm{ml} / \mathrm{min}$ ). Also there were significant difference between group A and group $\mathrm{B}$ in measuring of PImax.

The effect of IMT combined with other forms of exercise was examined in three studies. Winklemann et al.,2009 who applied his study on twenty-four patients with CHF and IMW (maximal inspiratory pressure $70 \%$ of predicted) were randomly assigned to a 12-week program of AE plus IMT $(\mathrm{AE}+\mathrm{IMT}, \mathrm{n}=$ 12 ) or to $A E$ alone (AE, $n=12)$. Before and after intervention, the following measures were obtained: maximal inspiratory muscle pressure (PImax), peak oxygen uptake $\left(\mathrm{VO}_{2}\right.$ peak), peak circulatory power, oxygen uptake efficiency slope, ventilatory efficiency, ventilatory oscillation, oxygen uptake kinetics during recovery $\left(\mathrm{T} 1 / 2 \mathrm{VO}_{2}\right)$ demonstrated that $30 \mathrm{~min}$ of daily threshold IMT performed at 30\% of MIP combined with aerobic exercise (cycling for $20-45 \mathrm{~min}, 3 \times /$ week, at the first ventilatory threshold) resulted in additional significant improvements in MIP, peak $\mathrm{VO}_{2}$, circulatory power, efficiency of ventilation and oxygen uptake and recovery oxygen kinetics .

Similar results were observed by Laoutaris et al., 2012. who found that 20 min of Tire trainair IMT performed at $60 \%$ of maximal inspiratory pressure/ sustained maximal inspiratory pressure ( MIP/SMIP) combined with $15 \mathrm{~min}$ of resistance training (three exercise sets of 10-12 dynamic quadriceps repetitions at $50 \%$ of $1 \mathrm{RM}$ as well as two exercise sets of 10-12 upper extremity repetitions using 1-2kg dumbbells) and 20-30min of aerobic exercise (cycling, 3x/week, at $70-80 \%$ of peak heart rate) resulted in additional significant improvements in the SMIP, quadriceps strength and endurance, exercise time, circulatory power, dyspnea and Quality of Life (QoL).

Cahalin et al. 2001 studied the combined IMT with expiratory muscle training (EMT) and compared combined IMT and EMT to cycle ergometry training (CET) and found results indicative of the specificity of 
training principle in that combined IMT and EMT improved IME and pulmonary function while CET improved cycling duration. Similar improvements in respiratory muscle strength, 6-MWT distance, estimated peak $\mathrm{VO} 2$, symptoms and QoL were observed in the combined IMT and EMT group and CET group . Administration of IMT combined with other modes of exercise appears promising for HF patients.

Dall'Ago et al ., 2006 found that in patients with $\mathrm{CHF}$ and inspiratory muscle weakness, IMT results in marked improvement in inspiratory muscle strength, as well as improvement in functional capacity, ventilator response to exercise, recovery oxygen uptake kinetics, and QoL . Thirty-two patients with $\mathrm{CHF}$ and weakness of inspiratory muscles (maximal inspiratory pressure [PImax]_70\% of predicted) were randomly assigned to a 12 -week program of inspiratory muscle training (IMT, 16 patients) or to a placebo-inspiratory muscle training (P-IMT, 16 patients). The following measures were obtained before and after the program: PImax at rest and $10 \mathrm{~min}$ after maximal exercise; peak oxygen uptake, circulatory power, ventilatory oscillations, and oxygen kinetics during early recovery $\left(\mathrm{V}^{\cdot} \mathrm{O}_{2} / \mathrm{t}_{-}\right.$ slope); 6-min walk test; and quality of life scores. The IMT resulted in a $115 \%$ increment PImax, $17 \%$ increase in peak oxygen uptake, and 19\% increase in the 6-min walk distance. Likewise, circulatory power increased and ventilator oscillations were reduced. The V' O2/t_slope (oxygen uptake during recovery) was improved during the recovery period, and quality of life scores improved.

\section{Conclusion:}

This study demonstrates that the addition of IMT to AE results in improvement in ventilatory responses to exercise in selected patients with CHF and IMW.

\section{Conflict of interest:}

Authors have declared that no conflict of interests exists.

\section{References}

1. Agnew N(2010): Preoperative cardiopulmonary exercise testing. Continuing Education in Anesthesia, Critical Care and Pain; 10: 33-37.

2. Badr NM, Mohamed RA and El-Azab AM (2014): Effect of Inspiratory Muscle Training on Maximal Inspiratory Pressure in Patients with Congestive Heart Failure Global Journal of Medical research; 14(3), Version 1.0. 
3. Bosnak-Guclu M, Arikan H, Savci S, Inal-Ince D, Tulumen E, Aytemir K and Tokgo“zoglu L (2011): Effects of inspiratory muscle training in patients with heart failure. Respiratory Medicine; 105: 1671-1681.

4. Cahalin L, Wagenaar R, Dec GW and Semigran MJ (2001): Endurance training in heart failure - a pilot study of the effects of cycle versus ventilatory muscle training. Circulation; 104(17):450-453 .

5. Chatterjee1 S, Sengupta S, Nag M, Kumar P, Goswamil S and Rudra A (2013): Cardiopulmonary Exercise Testing: A Review of Techniques and Applications .J Anesth Clinic Res; 4:7.

6. Crowe J and Bradley C (2008): The effectiveness of incentive spirometry with physical therapy for high risk patients after coronary artery bypass surgery. Phys Tnco; 17:260-268.

7. Dall'Ago P, Chiappa GR, Guths H, et al (2006): Inspiratory muscle training in patients with heart failure and inspiratory muscle weakness. A randomized trial. J Am Coll Cardiol; 47:757763.

8. Kennedy K , Adams J, Cheng D, and Berbarie Ra F (2012) : High-intensity track and field training in a cardiac rehabilitation program : Proc (Bayl Univ Med Cent) ;25(1):34-36

9. Laoutaris ID, Adamopoulos S, ManginasA et al (2012): Benefits of combined aerobic/ resistance/inspiratory training in patients with chronic heart failure. A complete exercise model? A prospective randomized study. Int $\mathbf{J}$ Cardiol doi:10.1016/j.ijcard.2012.05.019.
10. Piepoli MF, Conraads V, Corra U , Dickstein $\mathrm{K}$, Francis DP, Jaarsma T , McMurray J , Pieske B , Piotrowicz E, Schmid JP, Anker S D, Solal1 AC, Filippatos GS , Hoes AW , Gielen S , Giannuzzi P and Ponikowski PP (2011): Exercise training in heart failure: from theory to practice. A consensus document of the Heart Failure Association and the European Association for Cardiovascular Prevention and Rehabilitation. European Journal of Heart Failure; 13: 347-357.

11. Stein R, Chiappa GR, Guths H, et al . (2009): Inspiratory muscle training improves oxygen uptake efficiency slope in patients with heart failure and inspiratory muscle weakness. J Cardiopulm Rehabil Prev ; 23(3): 29-36.

12. Thayse LA, Vanessa RE, Illia N, Dantasflorentino 1, Mario E, Dourado J and Guilherme F (2012) :Effects of respiratory muscle training on respiratory muscle strength and heart rate variability in myotonic dystrophy patients type1.J Resp Cardiov Phy Ther; 1(1): 3-8.

13. Wong E, Selig S and Hare DL (2011): Respiratory muscle dysfunction and training in chronic heart failure. Heart Lung Circ; 20(5):289-294

14. Winkelmann ER, Chiappa GR, Lima CO, Viecili PR, Stein R and Ribeiro JP (2009): Addition of inspiratory muscle training to aerobic training improves cardiorespiratory responses to exercise in patients with heart failure and inspiratory muscle weakness. Am Heart J; 158 (5):768.e1-768.e7. 